MATEC Web of Conferences 22,05006 (2015)

DOI: $10.1051 /$ matecconf/ 20152205006

(C) Owned by the authors, published by EDP Sciences, 2015

\title{
A Study on the Expert System of Internal Medicine Diagnosis
}

\author{
Xiaohui Zhang \\ Central Laboratory, Langfang Health Vocational College, Langfang, Hebei, China
}

\begin{abstract}
In recent years, medical technologies are gradually developed and various kinds of expert diagnostic systems are gradually proposed. The expert system of internal medicine diagnosis is one of the important systems. In this paper, the design principle and functional modules of the expert system of internal medicine diagnosis are introduced. The calculation steps and the implementation procedures of computational calculation are also provided. With the expert system of BP neural network as the major research objects, this paper briefly explains the concept of BP neural network, provides the actual example with iron-deficiency anemia as the diagnostic object, and verifies the authenticity of this expert system diagnosis.
\end{abstract}

Keywords: internal medicine diagnosis; expert system; BP neural network; authenticity

\section{INTRODUCTION}

With the increasing progress of science and technology, those advanced technologies such as computers are becoming more and more widely-used in real life. In the medical field, diagnostic methods are not merely looking, listening, questioning and feeling the pulse. Nuclear magnetic monitoring and the CT technology have become commonly-used means in the medical field.

In 2014, Shenglan Chen et al discussed diagnostic problems of pulmonary embolism and pointed out clinical features of pulmonary embolism. Problems such as expiratory dyspnea, palpitation and fast heartbeat are primary diagnosis characteristics. The author chose Shaoxing Center Hospital and Taizhou Hospital as experimental sites so as to avoid the contingency of data as far as possible. Patients are taken as research objects, of which clinical characteristics, therapeutic methods and diagnosis essentials are research directions. Experimental results show that the death rate of patients treated by thrombolytic therapy is $6.25 \%$, the death rate of patients treated by anticoagulant therapy is $12.5 \%$, the death rate of patients treated by thrombolytic and anticoagulant therapy is $2.5 \%$, indicating that means combined with diagnostic and treatment should be an important diagnostic means of this disease.

In 2014, Yu Zhang et al analyzed the diagnosis of Alzheimer's disease and discussed the diagnostic method of neurology. Second Affiliated Hospital of Mudanjiang Medical College is taken as the site for sample data extraction. Diagnosis and treatment data of 40 patients is collected from Sept 2011 to Sept 2013. The average length of stay is 21 . There are 26 cases with healing effect, 13 cases with non-healing effect and one death case. The death cause is systemic failure. This disease is frequently seen in elderly people, including obvious hypophrenia, significant memory deterioration and clear aggressive behaviors. Treatment means mainly include drug treatment, daily care and non-drug treatment. The purpose of drug treatment is to improve memory and psychological status. Daily care mainly includes psychological guidance such as conversation and positive mental stimulation on nerve cells. The cause of the disease is relatively complex and there are no good means for radical cure at present. Comprehensive treatments are usually taken so as to improve the cognitive ability of patients, reduce bother of patients brought by the disease and improve the quality of life.

Guoyan Luo et al studied the internal medicine diagnosis problems of ulcerative colitis and analyzed the therapeutic schemes. The author chose a municipal hospital of Yunnan Province as the research site and conducted case analysis on 72 patients with ulcerative colitis. Among them, 55 patients are cured and 13 patients are improved in their physical conditions after being treated with internal western medicines. No patient has an untoward effect in the process of treatment. Thus, it suggests that internal medicine diagnosis and treatment are suitable for ulcerative colitis and the rate of untoward effect of patients is relatively low on the premise of applying this method.

This paper verifies the authenticity of the system from such aspects as algorithm design and algorithm implementation of internal medicine diagnosis system.

\section{INTERNAL MEDICINE DIAGNOSIS}

It is the key of internal medicine diagnosis that determines the disease type of a patient by observing the characteristics of the patient. As for digestive system diseases, symptoms such as stomachache, vomit and nausea are characteristics for diagnosis. As for diseases of respiratory system, pharyngitis, cough and fever are characteristics for diagnosis. As for circulation 
system diseases, there are substernal squeezing pain diagnostic characteristics of the disease. In addition, systemic diseases such as urinary system diseases and hematological system diseases have certain diagnostic characteristics. It is necessary to determine a specific disease after determining a certain systemic disease. For example, urinary system diseases include hyperthyroidism, diabetes and thyroid tumor. Further examination of the urinary system is shown in Figure 1.

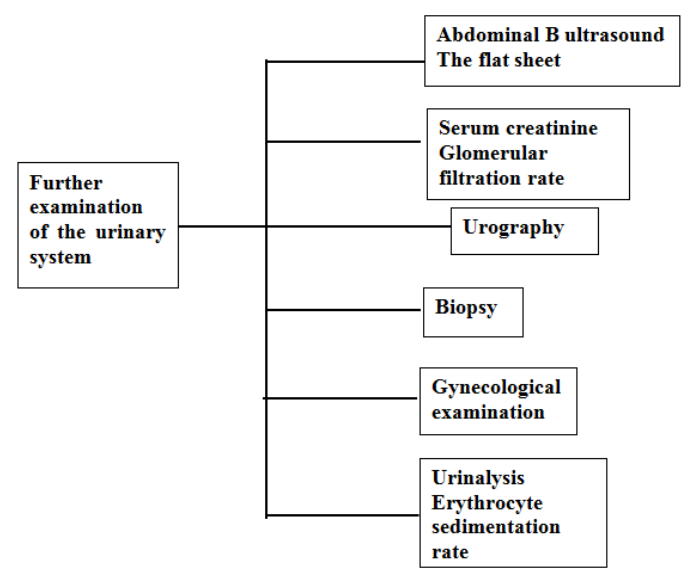

Figure 1. Further examination of the urinary system

\section{EXPERT SYSTEM}

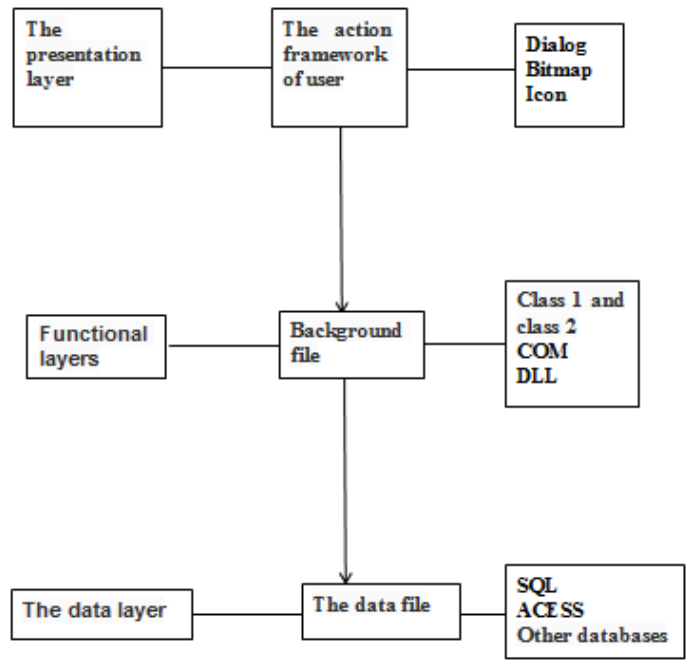

Figure 2. System design scheme

It is a system design concept to complete the system design with existing resources so as to finish diagnostic tasks of the expert system. Any kind of system development is not simply an application of a certain model. It is the comprehensive application of several kinds of models so that the system can effectively function. It can be seen from Figure 2 that the system includes models of three layers. And purposes of models are different so that diagnostic tasks of the

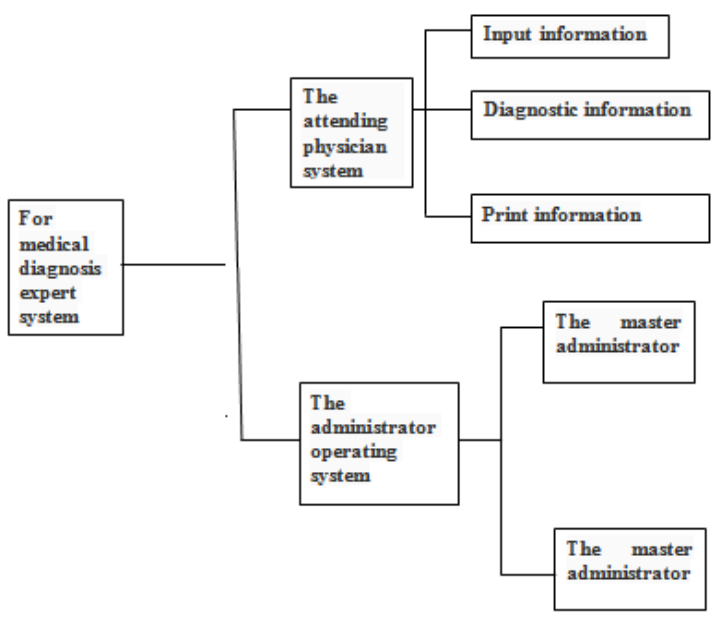

Figure 3. Internal medicine expert diagnostic system function module

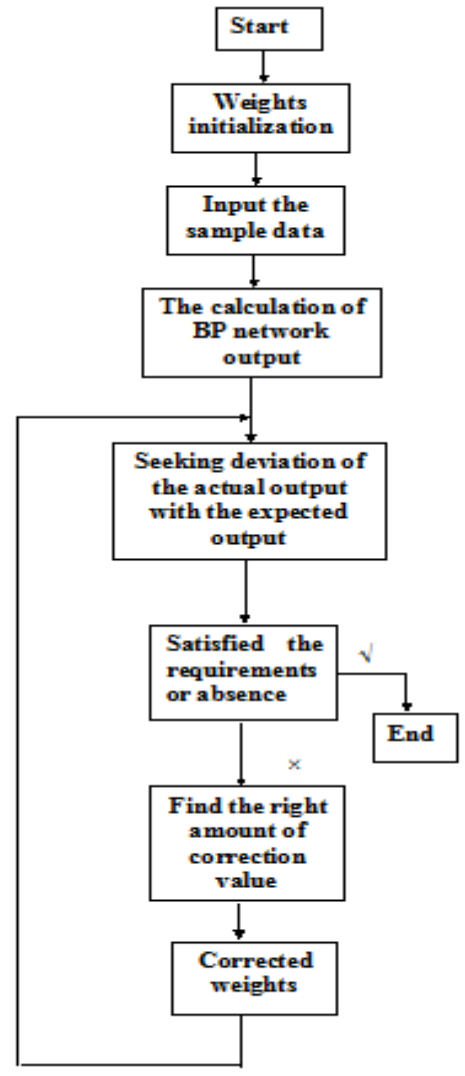

Figure 4. System algorithmic process

expert system can be completed.

It can be concluded from Figure 3 that the expert 
diagnostic system of internal medicine includes an administrator operating system and an attending physician system. Each system includes different functional modules. The administrator operating system includes information of administrators, information of physicians, parameters of the mathematical model and parameters of the system. The assistant administrator includes detailed information of physicians, parameters of the mathematical model and parameters of the system.

Figure 4 is the system algorithmic process. Initial data should be set before the execution of the algorithm such as calculation error, neural network layer and so on.

\section{BP NEURAL NETWORK}

\subsection{Concept of neural network model}

Neural network originates from neurobiology, the

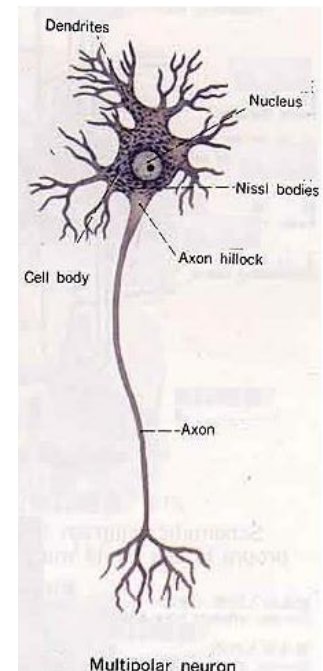

Figure 5. The structure of neurons

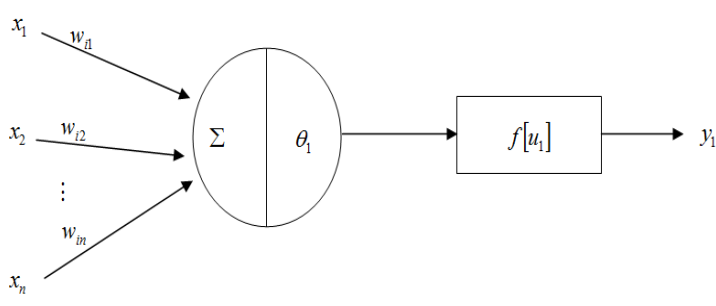

Figure 6. The schematic of mathematical models of neurons

computational process of which is similar to the reaction process of nerve cells in biology.

In the neural network, axon terminals in many dif- ferent neurons are able to enter dendrites of the same neuron to form a large number of synapses. Neurotransmitters released by all synapses of different sources are able to affect membrane potential changes of the same nerve cell. It reflects the ability of spatial information integration of nerve cells, which means that neurons can be integrated with input information of different sources on dendrites. According to this ability, the artificial neuron model is created by simu-

Table 1. Symbol descriptions of the mathematical model

\begin{tabular}{l|l}
\hline Symbols & Descriptions \\
\hline$x_{1}, x_{2}, \cdots, x_{n}$ & Input section of neurons, namely \\
& information released by the upper level \\
$\theta_{i}$ & Threshold of neurons \\
$y_{i}$ & Output of neurons \\
$f\left\lceil u_{1}\right\rceil$ & Excitation function \\
\hline
\end{tabular}

lating the reaction process of neurons, which is shown in Figure 6. Symbol descriptions are provided in Table 1.

$f\left[u_{1}\right]$ determines the output form of the threshold value $\theta_{i}$ based on the combined effect of input
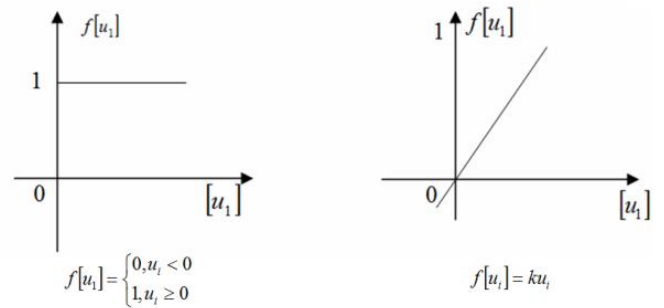

Figure 7. Typical excitation functions

$x_{1}, x_{2}, \cdots, x_{n}$. Images of two excitation functions are shown in Figure 7 . The model adopted in this paper uses the second excitation function.

Where,

$u_{i}=\sum_{j} w_{i j} x_{i}-\theta_{i}$

So

$y_{i}=f\left[u_{i}\right]=f\left(\sum_{j} w_{i j}-\theta_{i}\right)$

Formula (2) is the complete mathematical model expression of a single neuron.

\subsection{Calculation steps of the BP neural network model}

$B P$ neural network is a forward multilayer network calculated in the minimum mean square error. Sigmoid is adopted as the excitation function when the back propagation algorithm is applied in the feed-forward multilayer network. The recursion of network weight coefficient $w_{i j}$ is calculated in the following steps: 
(1) Assign $w_{i j}$. Assign $w_{i j}$ of each layer with a relatively small nonzero random number. Simultaneously, $w_{j n+1}=-\theta_{i}$. This model is operated through Matlab, so the assignment is random in the computer Thus, same program codes might have different results in different operating procedures

(2) Input sample value $x=\left(x_{1}, x_{2}, \cdots, x_{n}, 1\right)$ and correspond to expected output $y=\left(y_{1}, y_{2}, \cdots, y_{n}, 1\right)$.

(3) Calculate the output of each layer. As for the output $x_{i k}$ of the $i^{\text {th }}$ neuron in the $k^{\text {th }}$ layer,

$y_{i}^{k}=f\left[u_{i}^{k}\right]$

Where,

$u_{i}^{k}=\sum_{j} w_{i j} x_{j}^{k-1}-\theta_{i}^{k}$

In this formula, $x_{n+1}^{k-1}=1, \quad w_{i(n+1)}=-\theta$

(4) Calculate the error $d_{i}^{k}$ of each layer. As for the input layer, $k=m$, so

$d_{i}^{m}=x_{i}^{m}\left(1-x_{i}^{m}\right)\left(x_{i}^{m}-y_{i}^{m}\right)$

As for other layers,

$d_{i}^{k}=x_{i}^{k}\left(1-x_{i}^{k}\right)\left(\sum_{j} w_{i j} x_{j}^{k-1}-\theta_{i}^{k}\right)$

(5) Correct $w_{i j}$ and $\theta_{i}$, so

$w_{i j}(t+1)=w_{i j}(t)-\eta d_{i}^{k} x_{j}^{k-1}$

(6) It can be determined whether the requirements are satisfied in accordance with established criteria after the weight coefficient of each layer is calculated. If not, go back to the step (3). Otherwise, it will end the calculation.

\section{ALGORITHMIC EXAMPLES}

The design principle of the expert system of internal medicine diagnosis is briefly introduced mentioned earlier. Several simple practical algorithmic examples are listed as follows. The operating process of the expert system is introduced through the example of iron-deficiency anemia. For example, there are two

Table 2. Parameters of characteristic values

\begin{tabular}{|l|l|l|}
\hline Classification & Serum ferritin ug/L & Sideroblast \\
\hline \multirow{4}{*}{ Normal } & 9.80 & $10 \%$ \\
\cline { 2 - 3 } & 10.00 & $12 \%$ \\
\cline { 2 - 3 } & 11.58 & $13 \%$ \\
\cline { 2 - 3 } & 11.96 & $14 \%$ \\
\hline \multirow{4}{*}{ Abnormal } & 13.98 & $18 \%$ \\
\cline { 2 - 3 } & 14.96 & $26 \%$ \\
\cline { 2 - 3 } & 16.98 & $36 \%$ \\
\cline { 2 - 3 } & 19.68 & $45 \%$ \\
\hline
\end{tabular}

reference indexes of iron-deficiency anemia. One is serum ferritin and the other is sideroblast. The two characteristic values are first input and shown in Table 2.

Data in Table 2 is taken as the configuration parameters of the system. Figure 8 can be acquired through the algorithm simulation of the expert system with

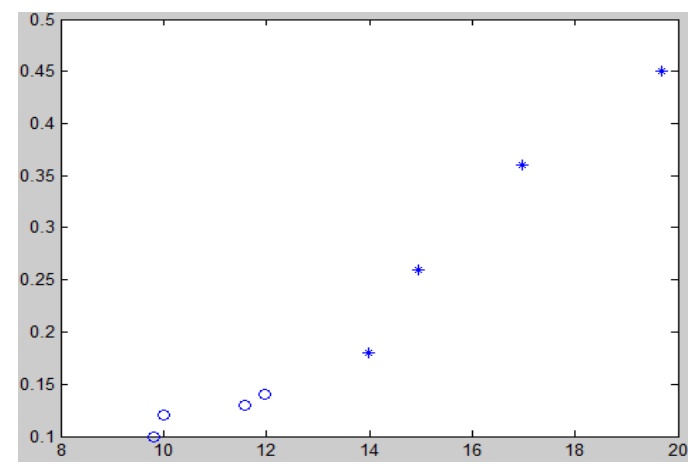

Figure 8. Parameter distribution

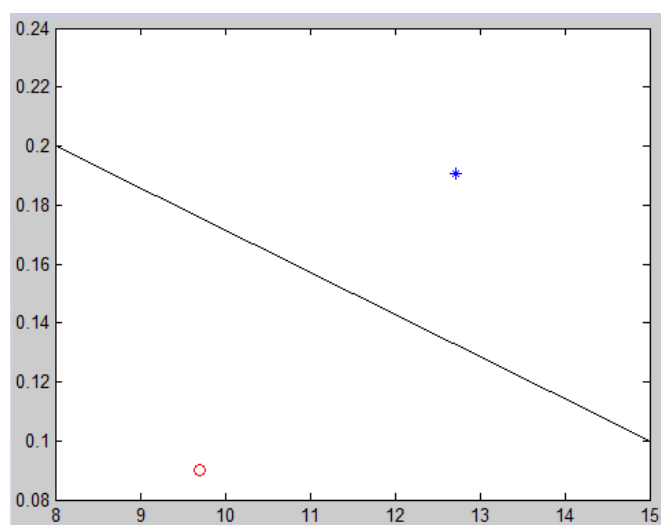

Figure 9. Judged results figure

\section{Matlab software.}

In Figure 8, "O" is the parameter of a normal person and "*" is the parameter of an abnormal person. It can be seen from the figure that there is an obvious boundary between parameters of normal and abnormal people. It can be determined that whether a subject has anemia by inputting parameters in the program. $\mathrm{Pa}$ rameters of patient $A$ are $(9.70,0.09)$ and parameters of patient $\mathrm{B}$ are $(12.68,0.24)$. Results are shown in Figure 9.

It can be seen from Figure 9 that $A$ is a healthy person while $\mathrm{B}$ is a patient with iron-deficiency anemia. The figure is in accordance with the actual results, reflecting the validity of this system. 


\section{CONCLUSION}

This paper applies BP neural network into the research on the expert system of internal medicine diagnosis and indicates the rationality and the validity of the expert system of internal medicine diagnosis from the angle of computer based on a brief introduction of the internal medicine diagnosis principle. However, applications of BP neural network have certain drawbacks. For example, a great deal of data needs to be analyzed if there are numerous factors for the determination of a certain disease. Practical situations cannot be reflected authentically and objectively due to the complex calculation process of the neural network model. Besides, the neural network needs to reasonably estimate the training error. Once the error is not reasonable, there might be incorrect calculation results. Therefore, initial values of parameters should be properly set for the expert diagnostic system with BP neural network as the application principle.

\section{REFERENCES}

[1] Wu, Y. 2010. Research and Implementation of the Expert System Based on Pathological Image Diagnosis. Wuhan: Wuhan University of Technology.

[2] Luo, G.Y. \& Kong, D.Q. 2014. An analysis on internal diagnosis and treatment of ulcerative colitis, Contemporary Medicine, (28): 77 .

[3] Chen, S.L., Lin, L., et al. 2014. An analysis on internal diagnosis and treatment of pulmonary embolism, Chinese Rural Health Service Administration, (7): 893.

[4] Zhang, Y. 2014. Neurology diagnosis and treatment of Alzheimer's disease, Inspection and Diagnosis, (5): 229.

[5] Zhou, Y.Z., et al. 2010. Mathematical Modeling, Shanghai: Tongji University Press.

[6] Wan, X.H. 2007. Probability Theory and Mathematical Statistics, Beijing: Science Press.

[7] Wang, X.Y., et al. 2010. Mathematical Modeling and Mathematical Experiment, Beijing: Science Press.

[8] Zhuo, J.W., et al. 2010. The Application of Matlab in Mathematical Modeling, Beijing: Beijing University of Aeronautics and Astronautics Press. 